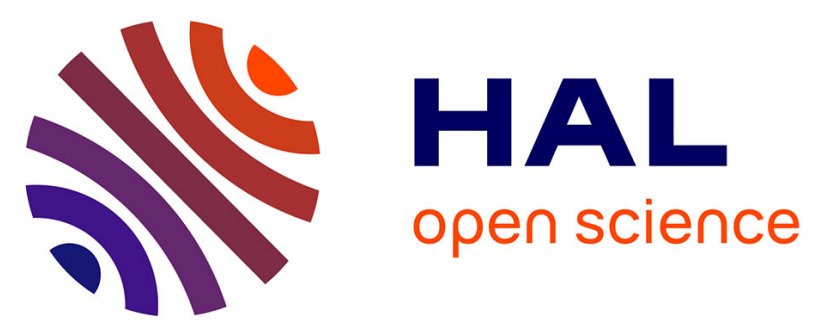

\title{
Resolution of Nonlinear Magnetostatic Problems With a Volume Integral Method Using the Magnetic Scalar Potential
}

Anthony Carpentier, Olivier Chadebec, Nicolas Galopin, Gérard Meunier, Bertrand Bannwarth

\section{To cite this version:}

Anthony Carpentier, Olivier Chadebec, Nicolas Galopin, Gérard Meunier, Bertrand Bannwarth. Resolution of Nonlinear Magnetostatic Problems With a Volume Integral Method Using the Magnetic Scalar Potential. IEEE Transactions on Magnetics, 2013, pp.1685-1688. 10.1109/TMAG.2013.2241750 . hal-00822687

\section{HAL Id: hal-00822687 https://hal.science/hal-00822687}

Submitted on 2 Feb 2021

HAL is a multi-disciplinary open access archive for the deposit and dissemination of scientific research documents, whether they are published or not. The documents may come from teaching and research institutions in France or abroad, or from public or private research centers.
L'archive ouverte pluridisciplinaire HAL, est destinée au dépôt et à la diffusion de documents scientifiques de niveau recherche, publiés ou non, émanant des établissements d'enseignement et de recherche français ou étrangers, des laboratoires publics ou privés. 


\title{
Resolution of Nonlinear Magnetostatic Problems With a Volume Integral Method Using the Magnetic Scalar Potential
}

\author{
Anthony Carpentier ${ }^{1}$, Olivier Chadebec ${ }^{1,2}$, Nicolas Galopin ${ }^{1}$, Gérard Meunier ${ }^{1}$, and Bertrand Bannwarth ${ }^{1}$ \\ ${ }^{1}$ Grenoble Electrical Engineering Laboratory, University of Grenoble, Cedex, France \\ ${ }^{2}$ GRUCAD/EEL/CTC/UFSC, Florianópolis, Brazil
}

\begin{abstract}
An integral method using the magnetic scalar potential to solve nonlinear magnetostatic problems is developed. This method uses the range interactions between magnetizable elements and it is particularly well suited to compute field in the air domain which do not need to be meshed. The collocation and Galerkin approaches are presented and compared to solve the nonlinear magnetostatic equation. Both methods need the construction of full interaction matrices which may be computed with analytical formulae. A Newton-Raphson method, in which the interaction matrix must be built at each solver iteration, is used to solve the nonlinear formulation. A modified fixed point scheme, in which the interaction matrix is built only once, is also proposed. 3-D numerical examples are given and results of the different methods are compared.
\end{abstract}

Index Terms-Integral method, magnetostatics, nonlinear.

\section{INTRODUCTION}

$\mathbf{F}$ OR devices with a huge volume free space compared to the active structure or high ratio between the object geometries, the finite element method leads to problems of accuracy and convergence to the solution [1]. In such case, integral methods can be attractive alternatives [2], [3]. Integral formulations of the magnetostatic field problems are particularly advantageous for the numerical solution of open-boundary problems which include ferromagnetic materials since only the active regions containing these materials need to be discretized. This paper gives in the first part a magnetostatic formulation in the framework of the integral volume methods in which the collocation and Galerkin methods are used to solve the magnetostatic equations. The computation of the source potential and of the integrals to built the interaction matrix are so presented in this part. The second part proposes the Newton-Raphson and modified fixed point method to solve the nonlinear formulation. The last one presents the results obtained for different applications.

\section{VOLUME INTEGRAL MethoD}

\section{A. Formulation}

Let us consider the following magnetostatic problem. A three-dimensional simply connected region $\Omega_{f}$ is filled with isotropic ferromagnetic material with the known linear magnetic susceptibility $\chi$. Primary sources of magnetic field in which currents flows are associated to the region $\Omega_{j}$. Both regions, $\Omega_{f}$ and $\Omega_{j}$, are disposed in free space $\Omega_{0}$ so that these regions do not overlap, $\Omega=\Omega_{f} \cup \Omega_{j} \cup \Omega_{0}$. We note $\Gamma$ the boundary between the ferromagnetic material $\Omega_{f}$ and the free space $\Omega_{0}$.

The ferromagnetic behavior law is defined by

$$
\mathbf{M}(\mathbf{r})=\chi(\mathbf{r}, \mathbf{H}) \mathbf{H}(\mathbf{r})
$$

where $\mathbf{M}(\mathbf{r})$ is the magnetization, $\mathbf{H}(\mathbf{r})$ the magnetic field at point of coordinates $r$. At any point of $\Omega$, the magnetic field is a sum of the reduced magnetic field created by the ferromagnetic material $\mathbf{H}_{\text {red }}(\mathbf{r})$ and the magnetic source field created by currents flows $\mathbf{H}_{0}(\mathbf{r})$ :

$$
\mathbf{H}(\mathbf{r})=\mathbf{H}_{\text {red }}(\mathbf{r})+\mathbf{H}_{0}(\mathbf{r}) .
$$

The simply connected region $\Omega_{f}$ containing no current sources, the following volume integral equation [4] using the total scalar potential $\Phi$ is considered:

$$
\Phi(\mathbf{r})+\frac{1}{4 \pi} \int_{\Omega_{f}} \chi\left(\mathbf{r}^{\prime}, \mathbf{H}\right) \frac{\nabla \Phi\left(\mathbf{r}^{\prime}\right) \cdot\left(\mathbf{r}-\mathbf{r}^{\prime}\right)}{\left|\mathbf{r}-\mathbf{r}^{\prime}\right|^{3}} \mathrm{~d} \Omega^{\prime}=\Phi_{0}(\mathbf{r})
$$

where $\Phi_{0}$ is the scalar potential deriving whose the magnetic field $\mathbf{H}_{0}$ produced by sources derives. Only the domain $\Omega_{f}$ is meshed and the potential $\Phi$ is approximated with first order nodal shape functions:

$$
\Phi \approx \sum_{i} \Phi_{i} \alpha_{i}
$$

where $\Phi_{i}$ and $\alpha_{i}$ are respectively the degree of freedom of $\Phi$ and the first order nodal shape function associated to the $i$ th mesh node.

Both resolution methods are used to solve (3). The first one is a collocation method at mesh nodes and it leads to solve a system of algebraic equations of the form

$$
([I]+[A]) \boldsymbol{\Phi}=\boldsymbol{\Phi}_{0}
$$

with

$$
\begin{aligned}
I_{i j} & =\delta_{i j} \\
A_{i j} & =\frac{1}{4 \pi} \int_{\Omega_{f}} \chi\left(\mathbf{r}^{\prime}, \mathbf{H}\right) \frac{\nabla \alpha_{j}\left(\mathbf{r}^{\prime}\right) \cdot\left(\mathbf{r}_{i}-\mathbf{r}^{\prime}\right)}{\left|\mathbf{r}_{i}-\mathbf{r}^{\prime}\right|^{3}} \mathrm{~d} \Omega^{\prime} .
\end{aligned}
$$

The weak formulation associated to (3) is

$$
\begin{aligned}
& \int_{\Omega_{f}} \boldsymbol{\alpha} \Phi_{0}(\mathbf{r}) \mathrm{d} \Omega=\int_{\Omega_{f}} \boldsymbol{\alpha} \Phi(\mathbf{r}) \mathrm{d} \Omega \\
& \quad+\frac{1}{4 \pi} \int_{\Omega_{f}} \boldsymbol{\alpha} \int_{\Omega_{f}} \chi\left(\mathbf{r}^{\prime}, \mathbf{H}\right) \frac{\nabla \Phi\left(\mathbf{r}^{\prime}\right) \cdot\left(\mathbf{r}-\mathbf{r}^{\prime}\right)}{\left|\mathbf{r}-\mathbf{r}^{\prime}\right|^{3}} \mathrm{~d} \Omega^{\prime} \mathrm{d} \Omega, \forall \boldsymbol{\alpha} .
\end{aligned}
$$

The second method is a Galerkin's method. Applied to the weak formulation (8) it leads to the algebraic equation system:

$$
([B]+[C]) \mathbf{\Phi}=\mathbf{D}
$$


with

$$
\begin{aligned}
B_{i j} & =\int_{\Omega_{f}} \alpha_{i}(\mathbf{r}) \alpha_{j}(\mathbf{r}) \mathrm{d} \Omega \\
C_{i j} & =\frac{1}{4 \pi} \int_{\Omega_{f}} \alpha_{i}(\mathbf{r}) \int_{\Omega_{f}} \chi\left(\mathbf{r}^{\prime}, \mathbf{H}\right) \frac{\nabla \alpha_{j}\left(\mathbf{r}^{\prime}\right) \cdot\left(\mathbf{r}_{i}-\mathbf{r}^{\prime}\right)}{\left|\mathbf{r}_{i}-\mathbf{r}^{\prime}\right|^{3}} \mathrm{~d} \Omega^{\prime} \mathrm{d} \Omega \\
D_{i} & =\int_{\Omega_{f}} \alpha_{i}(\mathbf{r}) \Phi_{0}(\mathbf{r}) \mathrm{d} \Omega .
\end{aligned}
$$

Once the problem solved, the magnetic field in the free space $\Omega_{0}$ is computed from the following relation:

$$
\mathbf{H}(\mathbf{r})=-\frac{1}{4 \pi} \sum_{e} \nabla\left(\int_{\Omega_{e}} \frac{\mathbf{M}_{e} \cdot\left(\mathbf{r}-\mathbf{r}^{\prime}\right)}{\left|\mathbf{r}-\mathbf{r}^{\prime}\right|^{3}} \mathrm{~d} \Omega_{e}\right)+\mathbf{H}_{0}(\mathbf{r})
$$

where $\mathbf{M}_{e}$ is the magnetization and $\Omega_{e}$ the domain of the $e$ th mesh element.

\section{B. Computation of the Source Potential}

The source field $\mathbf{H}_{0}$ can be expressed analytically for some coils geometries ([5] for example). Thus there are no direct analytical expressions for the source potential $\Phi_{0}$. A numerical computation is envisaged and the following minimization problem is considered:

$$
\min _{\Phi_{0}}\left(\left(\mathbf{H}_{0}+\nabla \Phi_{0}\right)^{2}\right)
$$

The weak formulation associated to the minimization problem (14) is

$$
\int_{\Omega_{f}} \nabla \boldsymbol{\alpha} \cdot \nabla \Phi_{0}(\mathbf{r}) \mathrm{d} \Omega=\int_{\Omega_{f}} \boldsymbol{\nabla} \boldsymbol{\alpha} \cdot \mathbf{H}_{0} \mathrm{~d} \Omega \quad \forall \boldsymbol{\alpha} .
$$

A finite element method is used on the weak form (15) and it leads to the resolution of an algebraic equation system:

$$
[E] \mathbf{\Phi}_{0}=\mathbf{F}
$$

with

$$
\begin{aligned}
E_{i j} & =\int_{\Omega_{f}} \nabla \alpha_{i} \cdot \nabla \alpha_{j} \mathrm{~d} \Omega \\
F_{i} & =-\int_{\Omega_{f}} \nabla \alpha_{i} \cdot \mathbf{H}_{0} \mathrm{~d} \Omega .
\end{aligned}
$$

This method needs a finite element method applied only to the active materials. However the computation of the source field at Gauss points could be expensive. A fast multipole method could so be used [6].

\section{Integrals Computation}

The (7), (11) and (13) need the computation of integrals with Green's kernels. These integrations take the form

$$
\begin{aligned}
& I_{1}(\mathbf{r})=\int_{\Omega_{e}} \frac{\mathbf{S} \cdot\left(\mathbf{r}-\mathbf{r}^{\prime}\right)}{\left|\mathbf{r}-\mathbf{r}^{\prime}\right|^{3}} \mathrm{~d} \Omega^{\prime} \\
& I_{2}(\mathbf{r})=\nabla \int_{\Omega_{e}} \frac{\mathbf{S} \cdot\left(\mathbf{r}-\mathbf{r}^{\prime}\right)}{\left|\mathbf{r}-\mathbf{r}^{\prime}\right|^{3}} \mathrm{~d} \Omega^{\prime}
\end{aligned}
$$

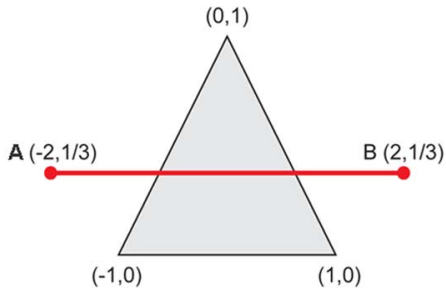

(a)

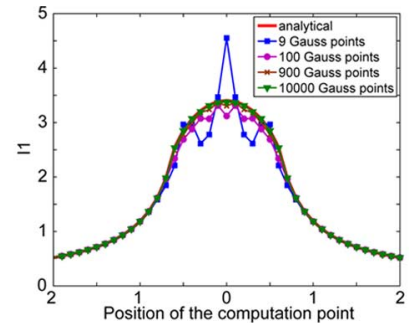

(b)
Fig. 1. Computation of the integral of the Green's kernel $I_{1}$ on an element. (a) Description of the integration element. (b) Results.

where $\mathbf{S}$ is an uniform field on the domain $\Omega_{e}$.

Using a vector identity and the Green-Ostrogradsky's theorem, (19) and (20) can be written as

$$
\begin{aligned}
& I_{1}(\mathbf{r})=\int_{\Gamma_{\epsilon}} \frac{\mathbf{S} \cdot \mathbf{n}}{\left|\mathbf{r}-\mathbf{r}^{\prime}\right|} \mathrm{d} \Gamma^{\prime} \\
& I_{2}(\mathbf{r})=\int_{\Gamma_{e}} \frac{(\mathbf{S} \cdot \mathbf{n})\left(\mathbf{r}-\mathbf{r}^{\prime}\right)}{\left|\mathbf{r}-\mathbf{r}^{\prime}\right|^{3}} \mathrm{~d} \Gamma^{\prime} .
\end{aligned}
$$

A classic approach to compute the previous integrals is a numerical integration with Gauss points. An alternative is to use analytical formulas. Several analytical expressions of the integrals (21) and (22) can be find in the literature for many geometries elements. Thereafter let us consider the analytical expression developed in [7] for any tetrahedral meshes. With (21) and (22) the domain integration can be reduced to the mesh faces. That is why we consider the integration on a triangle with a surface charge distribution equal to unity [Fig. 1(a)]. The computation points are along the segment $\mathrm{AB}$ [Fig. 1(a)] on the triangle plane passing through it. The computation of the integral of the Green's kernel $I_{1}$, using analytical expression and several numerical integrations with a varying number of Gauss points, is presented in Fig. 1(b).

When the computation point is too close to the integration domain, too many Gauss points are necessary to compute the integration of the Green's kernels with a good accuracy. Then analytical expressions are required. With the collocation approach, the assemblage of the interaction matrix $[A]$ of (5) uses a analytical expressions when the computation point is a node of the element integration. Otherwise a numerical integration with Gauss points is used. With the Galerkin approach, the assemblage of the interaction matrix $[C]$ of the (9) has two integrals. The first one uses a numerical integration with Gauss points. The second with the Green's kernel uses the analytical expression when the computation point is a Gauss point which is contained in the integration element. Otherwise a numerical integration with Gauss points is used. Four Gauss points are used on a tetrahedral mesh for numerical integrations with both approaches.

\section{NONLINEAR FORMULATION}

In a general case, the ferromagnetic behavior law of the material is nonlinear. A suitable solver is then required to solve the nonlinear formulation. Newton Raphson and modified fixed point schemes are investigated. In this section, let us consider the collocation approach and the GMRES method is used to solve the matrix systems.

\section{A. Newton-Raphson Scheme}

The Newton-Raphson scheme is a method to compute the zero of a function. A description of the method could easily be 


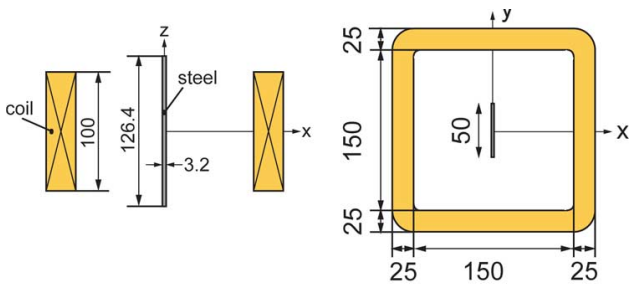

Fig. 2. Description of the steel plate problem.

found in the literature. The considered function coming from the behavior law (1) is

$$
F(\chi)=\chi(\mathbf{H})-\frac{\mathbf{M}(\mathbf{H})}{\mathbf{H}} .
$$

The following equation must be solved at each iteration:

$$
\begin{aligned}
\Phi^{k}(\mathbf{r})+\frac{1}{4 \pi} \int_{\Omega_{f}} \chi^{k}\left(\mathbf{r}^{\prime}, \mathbf{H}^{k-1}\right) \frac{\nabla \Phi^{k}\left(\mathbf{r}^{\prime}\right) \cdot\left(\mathbf{r}-\mathbf{r}^{\prime}\right)}{\left|\mathbf{r}-\mathbf{r}^{\prime}\right|^{3}} & \mathrm{~d} \Omega^{\prime} \\
& =\Phi_{0}(\mathbf{r})
\end{aligned}
$$

where $k$ is the iteration number. This scheme needs the computation of the interaction matrix at each iteration due to the local change of magnetic susceptibility in (24). Moreover the computation of the function derivative could be expensive.

\section{B. Modified Fixed Point Scheme}

The behavior law (1) is written

$$
\mathbf{M}(\mathbf{r}, \mathbf{H})=\chi_{\mathrm{FP}} \mathbf{H}(\mathbf{r})+\mathbf{S}(\mathbf{r}, \mathbf{H})
$$

where $\chi_{\mathrm{FP}}$ is the constant slope of the modified point scheme and $\mathbf{S}$ the nonlinear residual. The choice of the slop depends on the extreme values of the susceptibility $\chi_{\min }$ and $\chi_{\max }$ [8]. The optimal slop $\chi_{\mathrm{opt}}$ can be evaluated by [9]

$$
\chi_{\mathrm{opt}}=\frac{\chi_{\min }+\chi_{\max }}{2} .
$$

The fixed point can be found by iteratively updating the nonlinear residual. Using (25) in (3), the following equation must be solved at each iteration:

$$
\begin{aligned}
\Phi^{k}(\mathbf{r})+ & \frac{1}{4 \pi} \int_{\Omega_{f}} \chi_{\mathrm{FP}} \frac{\nabla \Phi^{k}\left(\mathbf{r}^{\prime}\right) \cdot\left(\mathbf{r}-\mathbf{r}^{\prime}\right)}{\left|\mathbf{r}-\mathbf{r}^{\prime}\right|^{3}} \mathrm{~d} \Omega^{\prime} \\
& =\Phi_{0}(\mathbf{r})+\frac{1}{4 \pi} \int_{\Omega_{f}} \frac{\mathbf{S}^{k}\left(\mathbf{r}^{\prime}, \mathbf{H}^{k-1}\right) \cdot\left(\mathbf{r}-\mathbf{r}^{\prime}\right)}{\left|\mathbf{r}-\mathbf{r}^{\prime}\right|^{3}} \mathrm{~d} \Omega^{\prime}
\end{aligned}
$$

where $k$ is the iteration number. The previous methods (5) and (9) are used to solve (27) at each iteration. The new value of the residual $\mathbf{S}$ at iteration $k$ is given by

$$
\mathbf{S}^{k+1}=\left(\mathbf{M}\left(\mathbf{H}^{k}\right)-\chi_{\mathrm{FP}} \mathbf{H}^{k}\right) .
$$

The algorithm is stopped when the norm of the difference of magnetic susceptibility between two iterations is lower than a criterion. This scheme enables the computation of a single interaction matrix during all the resolution process.

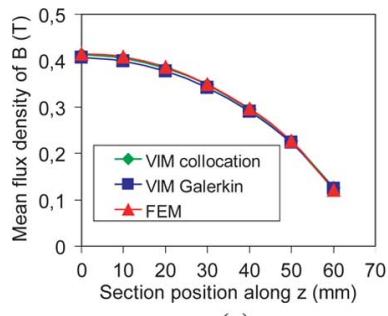

(a)

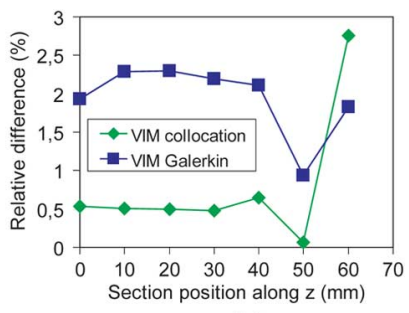

(b)
Fig. 3. Results of the steel plate problem. (a) Average magnetic flux density computed along the plate. (b) Relative difference with FEM.

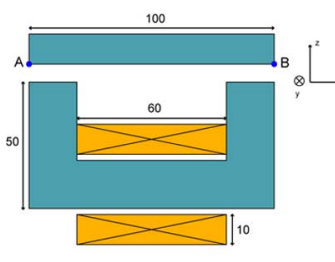

(a)

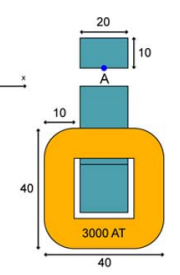

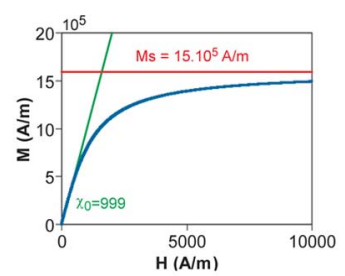

(b)
Fig. 4. Description of the contactor problem. (a) Geometry. (b) Behavior law.

\section{APPLICATIONS}

\section{A. Steel Plate Problem}

A steel plate with a linear behavior law is placed in a magnetic field generated by a coil (Fig. 2). The results obtained by the volume integral method with collocation and Galerkin approaches are compared with those obtained by the finite element method using the software Flux3D ${ }^{\circledR}[10]$. The evolutions of average flux density of the magnetic induction $\mathbf{B}$ along the $\mathbf{z}$ axis on the plate are computed with the three methods [Fig. 3(a)]. The relative difference with the FEM results are also presented in Fig. 3(b).

Both methods give comparable results to the finite element method. The Galerkin's approach seems undervalued the field. An assumption is that the use of Gauss points for the first integral (11) are not able to compute with accuracy the decreasing of the integral with the Green's kernel (11) when both integration domains are too close. Nevertheless, the collocation is less accurate on the geometry singularities due to the collocation method at nodes. The assemblage of the interaction matrix with collocation method is much faster than with Galerkin's one. That is why the collocation approach is preferred in order to model more complex applications like in the next parts.

\section{B. Contactor-Like Problem}

Let us consider the contactor-like problem described by Fig. 4(a). The ferromagnetic material has a nonlinear behavior law with an arctangent form presented in Fig. 4(b). The volume integral method with a collocation approach (3) is used to solve the magnetostatic field distribution. The previous modified fixed point and the Newton-Raphson methods are used to solve the nonlinear formulation. The value of 550 evaluated by (26) is assigned to the slope $\chi_{\mathrm{FP}}$ to ensure a good convergence with the modified fixed point method. The computed magnetostatic field distribution are presented Fig. 5. The performance of both methods to solve the nonlinear formulation are compared in Table I.

In this problem the material is few saturated so the resolution with the modified fixed point method converges relatively 


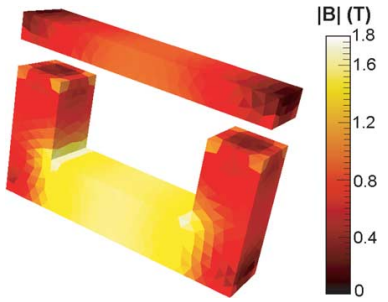

Fig. 5. Computed magnetic flux density for the contactor problem.

TABLE I

Performance of Both Methods to Solve the Nonlinear FORMULATION FOR A CONVERGENCE CRITERION EQUAL TO $1.10^{6}$

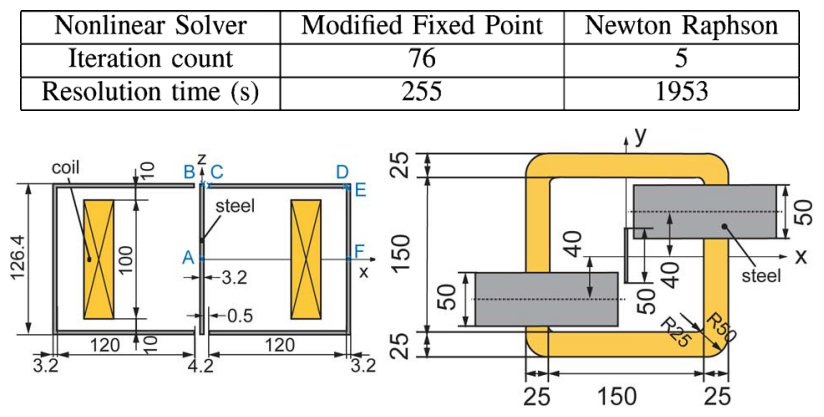

Fig. 6. Description of the team problem 13.

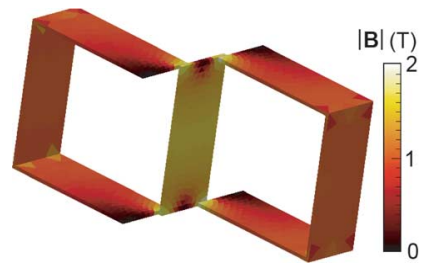

Fig. 7. Computed magnetic flux density for the team problem 13.

rapidly. Nevertheless this method requires much more iterations to converge than the Newton-Raphson one. Since only the right is actualized in (27), the duration of each iteration is short. That is why the time performance of modified fixed point method are better in this case.

\section{Team Problem 13}

In order to evaluate the performance of the volume integral method, the TEAM problem 13, described by Fig. 6, is considered. The curve of the magnetic behavior law of the steel can be found in [11]. It is known to be a nonlinear magnetostatic difficult problem. This problem is solved using the previous integral volume method with a collocation approach (3). The computed magnetostatic field distribution are presented in Fig. 7. The average flux densities of the magnetic induction $\mathbf{B}$ are compared with experimental results for various position along the plate and the yoke in Fig. 8.

As it is a 3-D problem and although the thicknesses are low, it is not possible to make the assumption of a tangential magnetization. Moreover, mesh refinement near the airgap and a sufficient number of elements in the thickness of yoke and plate are required to have a good discretization. The full storage of the interaction matrix of (5) or (9) limits the number of mesh elements. So a coarse mesh of 5716 tetrahedra is used. Moreover the material is highly saturated and particularly near the air gaps between the plate and the yokes. In this case, too many iterations are necessary to converge with the modified fixed point schema.

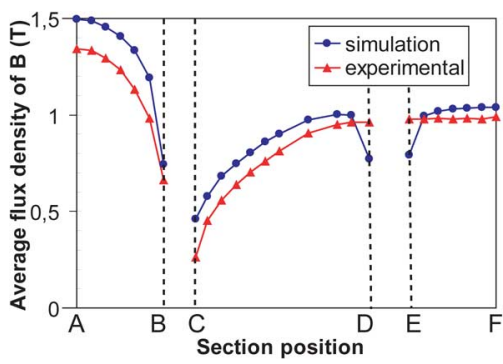

Fig. 8. Average magnetic flux density computed along the yoke and the plate.

The Newton-Raphson is preferred and it converges after 7 iterations with a criterion of $10^{-6}$. The results show a significant deviation between the simulation and experimental results. However the team problem 13 is a benchmark in which existing codes can give significantly different results [11]. That is why the results are hopeful considering the poor quality of the mesh. Compression techniques used on the interaction matrix would increase the number of processable elements.

\section{CONCLUSION}

A volume integral method using the magnetic scalar potential to solve nonlinear magnetostatic problems has been developed. The collocation and Galerkin's methods are presented to solve the equations. Both need to take care of the assemblage of interaction matrices. The collocation method is preferred in regard to necessary time for matrix assemblage. The resolution of the nonlinear formulation using the modified fixed point scheme required a unique assemblage of the interaction matrix. So, for any problems with a weakly saturated material, the modified fixed point scheme gives faster resolution than the classic Newton-Raphson one in which the interaction matrix is built at each iteration. For complex problems, compression and acceleration are required to make the volume integral method an efficient method to solve magnetostatic problems without the constraint of the air mesh.

\section{REFERENCES}

[1] H. L. Rakotoarison, V. Ardon, O. Chadebec, B. Delinchant, S. Guerin, and J. L. Coulomb, "Formal sensitivity computation of magnetic moment method," IEEE Trans. Magn., vol. 44, no. 6, pp. 1014-1017, 2008.

[2] A. Canova and M. Repetto, "Integral solution of nonlinear magnetostatic field problems," IEEE Trans. Magn., vol. 37, no. 3, pp. 1070-1077, 2001.

[3] W. Hafla, F. Groh, A. Buchau, and W. M. Rucker, "Magnetic field computation with integral equation method and energy-controlled relaxation," IEEE Trans. Magn., vol. 42, no. 4, pp. 719-722, 2006.

[4] A. Kalimov and M. Svedentsov, "Application of a hybrid integrodifferential method for analysis of thin magnetic shields," IEEE Trans. Magn., vol. 34, no. 5, pp. 2453-2456, 1998.

[5] L. K. Urankar, "Vector potential and magnetic field of current-carrying finite arc segment in analytical form, Part I: Filament approximation," IEEE Trans. Magn., vol. 16, no. 5, pp. 1283-1288, 1980.

[6] W. Hafla, A. Buchau, and W. M. Rucker, "Efficient computation of source magnetic potential," Adv. Radio Sci., vol. 4, pp. 59-63, 2006.

[7] C. Rubeck, J. P. Yonnet, H. Allag, B. Delinchant, and O. Chadebec, "Analytical calculation of magnet systems: Magnetic field created by charged triangles and polyhedra," IEEE Trans. Magn., to be published.

[8] F. I. Hantila, G. Preda, and M. Vasiliui, "Polarization method for static fields," IEEE Trans. Magn., vol. 36, no. 4, pp. 672-675, 2000.

[9] F. Ossart and V. Ionita, "Convergence de la méthode du point fixe modifiée pour le calcul de champ magnétique avec hystérésis," Eur. Phys. J. Appl. Phys., vol. 5, pp. 63-69, 1999.

[10] Flux3D®, Cedrat. [Online]. Available: http://www.cedrat.com.

[11] T. Nakate, N. Takahashi, and K. Fujiwara, "Summary of results for team workshop problem 13 (3-D nonlinear magnetostatic model)," COMPEL, vol. 14, no. 2/3, pp. 91-101, 1995. 